\title{
Aquatic Oligochaeta (Annelida: Clitellata) from State of São Paulo, Brazil: Diversity and Occurrence review
}

\author{
Guilherme Gorni $^{1,4}$, Douglas Fernando Peiró ${ }^{2} \&$ Nathalie Sanches ${ }^{3}$ \\ ${ }^{1}$ Programa de Pós Graduação em Desenvolvimento Regional e Meio Ambiente, Centro Universitário de \\ Araraquara, Rua Voluntários da Pátria, 1309, CEP 14801-320, Araraquara, SP, Brazil. \\ ${ }^{2}$ Departamento de Ciências Biológicas e da Saúde, Centro Universitário de Araraquara, Rua Voluntários da \\ Pátria, 1309, CEP 14801-320, Araraquara, SP, Brazil. \\ ${ }^{3}$ Centro Universitário de Araraquara, Rua Voluntários da Pátria, 1309, CEP 14801-320, Araraquara, SP, \\ Brazil. \\ ${ }^{4}$ Corresponding author: Guilherme Gorni, e-mail: grgorni@gmail.com
}

GORNI, G., PEIRÓ, D.F., SANCHES, N. Aquatic Oligochaeta (Annelida: Clitellata) from State of São Paulo, Brazil: Diversity and Occurrence review. Biota Neotropica. 15(1): e20140063. http://dx.doi.org/10. 1590/1676-06032015006314

\begin{abstract}
More than 5.000 species are described to Class Oligochaeta, and approximately 1650 aquatic species are recognized around the world. Knowledge regarding abundance and composition of limnic oligochaetes can be used as an indicator of water and sediment quality. Thus, this paper aims to summarize all published data on aquatic Oligochaeta from State of São Paulo (Brazil), in order to establish a cheklist of microdrili from the State. The list emphasizes the diversity of limnic oligochaetes and provides a general overview of their distribution and also ecological aspects. Were examined the bibliographical data on São Paulo aquatic Oligochaeta, including species catalogs and taxonomic and ecological literature. Were analyzed a total of 34 academic researches, 32 journal papers and two taxonomic catalogs. This analysis resulted in a list of 75 valid species, distributed in 24 genera and five families. This amount of taxa corresponds to $4.5 \%$ of the aquatic Oligochaeta worldwide valid species, and about $87 \%$ of the Brazilian species.
\end{abstract}

Keywords: Limnic oligochaetes, Species list, Occurrence, São Paulo.

GORNI, G., PEIRÓ, D.F., SANCHES, N. Oligochaeta aquático (Annelida: Clitellata) do Estado de São Paulo: Revisão da Diversidade e Ocorrência. Biota Neotropica. 15(1): e20140063. http://dx.doi.org/10.1590/ 1676-06032015006314

Resumo: Mais de 5.000 espécies são descritas para a Classe Oligochaeta e aproximadamente 1650 espécies aquáticas são reconhecidas em todo o mundo. O conhecimento sobre a composição e abundância de oligoquetos límnicos pode ser um indicador de qualidade da água e/ou sedimento. Assim, este trabalho teve como objetivo reunir todas as informações publicadas sobre Oligochaeta aquático do Estado de São Paulo (Brasil), a fim de estabelecer uma lista de táxons de microdrili registrados. A lista destaca a diversidade de oligoquetos límnicos e fornece uma visão geral de sua distribuição e aspectos ecológicos. Para isso, foram examinados dados bibliográficos de Oligochaeta aquáticos em São Paulo, incluindo catálogos de espécies, bem como artigos sobre taxonomia e de ecologia. Foram analisados ??o total de 34 trabalhos acadêmicos, sendo 32 artigos e dois catálogos taxonômicos. Esta análise resultou em uma lista de 75 espécies válidas, distribuídas em 24 gêneros e cinco famílias. Este montante corresponde a 4,5\% das espécies de Oligochaeta aquático registradas no mundo, e aproximadamente $87 \%$ das espécies brasileiras. Palavras-chave: Oligoquetos límnicos, Lista de espécies, Ocorrência, São Paulo.

\section{Introduction}

The Class Oligochaeta is included into the Phylum Annelida, together with Hirudinea and Polychaeta. The main characteristic of the phylum is the metameric body construction. It means the body is subdivided in similar segments (or "rings") arranged along the antero-posterior axis of the body (Righi 2002).

More than 5.000 species are described to Oligochaeta. The majority of them are terrestrial earthworms (megadrile). Being relatively low the number of freshwater, semi terrestrial or marine forms (microdrile). Approximately 1650 aquatic Oligochaeta are recognized as valid species around the world (Martin et al. 2008). In Brazil the number of valid species is around 86, excluding the Enchytraeidae Family (Christoffersen 2007). In continental aquatic environments the abundance and composition of Oligochaeta can be an indicator for water and sediment quality (Lafont 1984, Marchese \& Drago 1999, Alves \& Strixino 2000). 
The knowledge about the Brazilian freshwater Oligochaeta is very fragmented and incomplete, even these organisms being very common in continental aquatic environments (Timm et al. 2001). However, the publication of books in the eighties specifically focused on the identification of oligochaetes in Brazil and South America (Righi 1984, Brinkhurst \& Marchese 1989, respectively) - encouraged researches as Takeda (1999, 2001), Montanholi-Martins \& Takeda (1999, 2001), Alves \& Lucca (2000), Alves \& Strixino (2000), Pamplin et al. (2005), Alves et al. (2006, 2008), Gorni \& Alves (2008a, 2008b, 2012), Martins \& Alves (2010) and Rodrigues et al. (2013a, 2013b), which have broadened the knowledge on taxonomic and ecological aspects of these worms in Brazil.

The objective of this paper is to summarize all published data on aquatic Oligochaeta from State of São Paulo (Brazil), in order to establish a checklist of known microdrili taxa from the State. The list emphasizes the diversity of limnic oligochaetes and provides a general overview of their distribution and also ecological aspects.

\section{Material and methods}

Were examined the bibliographical data on São Paulo aquatic Oligochaeta, including species catalog, and taxonomic and ecological literature. The literature was surveyed by searching academic databases (Scopus, Scielo, and Google Scholar), and the scientific publications with reports of limnic oligochaetes. Aiming covering the greatest number of publications, were consulted academic papers published from 1942 through 2014.

\section{Results and discussion}

A total of 34 studies about São Paulo aquatic Oligochaeta were analyzed, including 32 journal papers and 2 taxonomic catalogs. The list of taxa includes 75 valid species, distributed in 24 genera and five families. This amount of species corresponds to $4.5 \%$ of the aquatic Oligochaeta worldwide valid species, and about $87 \%$ of the Brazilian species (Christoffersen 2007, Martin et al. 2008). This checklist is shown below, divided into families, genera and species, following alphabetical order.

\section{Checklist and classification}

Aelosomatidae

Aelosoma aerum Marcus, 1944

Records: ecological remarks

São Paulo: Serra da Cantareira streams associated with the bryophyte Dumortiera sp. (Marcus 1944).

\section{Aelosoma corderoi Du Bois Reymond Marcus, 1944}

Records:

São Paulo: Tietê River (Du Bois Reymond Marcus 1944).

Aelosoma evelinae Marcus, 1944

Records: ecological remarks

São Paulo: Pinheiros River, sediment (Marcus 1944).

Aelosoma gertae Marcus, 1944

Records: ecological remarks

São Paulo: associated with the aquatic macrophyte

Eichhornia crassipes (Marcus 1944).

Aelosoma headleyi Beddard, 1888

Records: ecological remarks

São Paulo: associated with the aquatic macrophyte Eichhornia crassipes (Marcus 1944).
Aelosoma hemprichi Ehrenberg, 1831

Records: ecological remarks

São Paulo: University of São Paulo Campus associated with the Bromeliaceae (Marcus 1944).

Aelosoma sawayai Marcus, 1944

Records:

São Paulo and Mogi das Cruzes (Marcus 1944).

Aelosoma travancorense Aiyer, 1926

Records:

São Paulo, Mogi das Cruzes and Pirassununga (Marcus 1944).

\section{Aelosoma viride Stephenson, 1911}

Records: ecological remarks

São Paulo: Pinheiros River tributaries, sediment and associated with the Bromeliaceae (Marcus 1944).

Alluroididae

Brinkhurstia americana (Brinkhurst, 1964)

Records: ecological remarks

Araraquara: Pinheirinho and Santa Clara Streams, sediment (Alves \& De Lucca 2000). Américo Brasiliense: Anhumas Stream Dam, sediment (Corbi \& Trivinho-Strixino 2002). Salesópolis and Bariri: Tietê River, sediment of Ponte Nova and Bariri Dams (Pamplin et al. 2005). Araraquara: Pinheirinho Stream, sediment (Alves et al. 2006).

Naididae

Allodero lutzi (Michaelsen, 1926)

Dero (Allodero) lutzi, Righi 1984: 26-27.

Records:

São Paulo (Righi 1984).

Allonais chelata (Marcus, 1944)

Records: ecological remarks

Américo Brasiliense: Anhumas Stream Dam, sediment (Corbi \& Trivinho-Strixino 2002). Brotas and Américo Brasiliense: Lagoa Dourada and Anhumas Stream Dams, associated with aquatic macrophytes (Alves \& Gorni 2007).

Allonais inaequalis (Stephenson, 1911)

Records: ecological remarks

Araraquara: Pinheirinho Stream, sediment (Alves et al. 2006), and Cruzes Stream, associated with the gastropod Pomacea bridgesii (Gorni \& Alves 2006). Brotas and Américo Brasiliense: Lagoa Dourada and Anhumas Stream Dams, associated with aquatic macrophytes (Alves \& Gorni 2007), and in Lagoa Dourada Dam, associated with the sponge Metania spinata (Gorni \& Alves 2008a).

Allonais paraguayensis paraguayensis (Michaelsen, 1905)

Nais paraguayensis, Marcus 1943: 23-31.

Allonais paraguayensis, Correia \& Trivinho-Strixino 1998: 41; Trivinho-Strixino et al. 2000: 533; Alves \& De Lucca 2000: 115; Corbi \& Trivinho-Strixino 2002: 38; Pamplin et al. 2005: 67; Janke \& Trivinho-Strixino 2007: 112; Gorni \& Alves 2008b: 163; Gorni \& Alves 2012: 107.

Allonais lairdi, Pamplin et al. 2005: 67.

Records: ecological remarks

São Paulo: Pinheiros River tributaries, sediment and Pirassununga: Mogi-Guaçu River and Cachoeira de Emas Reservoir, sediment (Marcus 1943). Luiz Antônio: MogiGuaçu River, associated with the aquatic macrophyte Scirpus cubensis (Correia \& Trivinho-Strixino 1998, Trivinho-Strixino et al. 2000). Araraquara: Pinheirinho and Santa Clara Streams, sediment (Alves \& De Lucca 2000). Américo Brasiliense: Anhumas Stream Dam, sediment (Corbi \& Trivinho-Strixino 2002). Salesópolis and Bariri: Tietê River, Ponte Nova and 
Bariri Dams, sediment (Pamplin et al. 2005). São Carlos: Fazzari Stream, leaf litter (Janke \& Trivinho-Strixino 2007). Campos do Jordão: Galharada Stream, sediment and leaf litter (Gorni \& Alves 2008b, Gorni \& Alves 2012).

Amphichaeta leydigi Tauber, 1879

Records:

Ribeirão Grande: Intervales State Park Streams (Alves et al. 2008).

Aulodrilus limnobius Bretscher, 1899

Records: ecological remarks

São Paulo: Pinheiros River, sediment (Marcus 1944). Ribeirão Grande: Intervales State Park Streams (Alves et al. 2008). Campos do Jordão: Galharada and Serrote Streams, sediment and leaf litter (Gorni \& Alves 2008b, Gorni \& Alves 2012).

Aulodrilus pigueti Kowalewski, 1914

Records: ecological remarks

Luiz Antônio: Mogi-Guaçu River, sediment (Alves \& Strixino 2000). Luiz Antonio: Mogi-Guaçu River, sediment (Alves \& Strixino 2003).

\section{Aulophorus borellii (Michaelsen, 1900)}

Dero (Aulophorus) borellii, Correia \& Trivinho-Strixino 1998: 41; Trivinho-Strixino et al. 2000: 533; Alves \& De Lucca 2000: 115 .

\section{Records: ecological remarks}

Luiz Antônio: Mogi-Guaçu River, associated with the aquatic macrophyte Scirpus cubensis (Correia \& TrivinhoStrixino 1998, Trivinho-Strixino et al. 2000). Araraquara: Pinheirinho and Santa Clara Streams, sediment (Alves \& De Lucca 2000).

\section{Aulophorus carteri Stephenson, 1931}

Dero (Aulophorus) carteri, Correia \& Trivinho-Strixino 1998: 41; Trivinho-Strixino et al. 2000: 533.

\section{Records: ecological remarks}

Pirassununga: Mogi-Guaçu River, Cachoeira de Emas Reservoir, sediment (Marcus 1943). Luiz Antônio: MogiGuaçu River, associated with aquatic macrophytes, Scirpus cubensis (Correia \& Trivinho-Strixino 1998, Trivinho-Strixino et al. 2000).

\section{Aulophorus costatus Du Bois-Reymond Marcus, 1944}

Dero (Aulophorus) costatus, Alves \& Gorni 2007: 409; Gorni \& Alves 2008a: 262.

\section{Records: ecological remarks}

Américo Brasiliense: Anhumas Stream Dams, associated with aquatic macrophytes (Alves \& Gorni 2007). Brotas: Lagoa Dourada Dam, associated with the sponge Metania spinata (Gorni \& Alves 2008a).

Aulophorus furcatus (O. F. Müller, 1774)

Dero (Aulophorus) furcatus, Pamplin et al. 2005: 67; Gorni \& Alves 2006: 1060; Suriani et al. 2007: 420-421.

\section{Records: ecological remarks}

São Paulo: sediment of Pinheiros and Tietê Rivers tributaries (Marcus 1943). Salesópolis and Bariri: Tietê River, Ponte Nova and Bariri Dams, sediment (Pamplin et al. 2005). Araraquara: Cruzes Stream, associated with the gastropod Pomacea bridgesii (Gorni \& Alves 2006). Barra Bonita: Tietê River, Barra Bonita Reservoir, sediment (Suriani et al. 2007).

Aulophorus hymanae (Naidu, 1962)

Dero (Aulophorus) hymanae, Suriani et al. 2007: 420-421.

Records: ecological remarks

Promissão: Tietê River, sediment of Promissão Reservoir, Buritama: Tietê River, Nova Avanhandava Reservoir, sediment.
Pereira Barreto: Tietê River, Três Irmãos Reservoir, sediment (Suriani et al. 2007)

Aulophorus lodeni (Brinkhurst, 1986)

Dero (Aulophorus) lodeni, Correia \& Trivinho-Strixino 1998: 41; Trivinho-Strixino et al. 2000: 533; Corbi \& TrivinhoStrixino 2002: 38; Pamplin et al. 2005: 67; Suriani et al. 2007: 420-421.

\section{Records: ecological remarks}

Luiz Antônio: Mogi-Guaçu River, associated with the aquatic macrophyte Scirpus cubensis (Correia \& TrivinhoStrixino 1998, Trivinho-Strixino et al. 2000). Américo Brasiliense: Anhumas Stream Dam, sediment (Corbi \& Trivinho-Strixino 2002). Salesópolis and Bariri: Tietê River, Ponte Nova and Bariri Dams, sediment (Pamplin et al. 2005). Barra Bonita: Tietê River, Barra Bonita Reservoir, sediment. Ibitinga: Tietê River, Ibitinga Reservoir, sediment. Promissão: Tietê River, Promissão Reservoir, sediment. Buritama: Tietê River, Nova Avanhandava Reservoir, sediment. Pereira Barreto: Tietê River, Três Irmãos Reservoir, sediment (Suriani et al. 2007).

Aulophorus superterrenus Michaelsen, 1912

Records: ecological remarks

São Paulo and Campinas: associated with the epiphytic Vriesea sp. (Bromeliaceae) (Marcus 1943).

\section{Bothrioneurum americanum Beddard, 1894}

Records: ecological remarks

Salesópolis and Bariri: Tietê River, Ponte Nova and Bariri Dams, sediment (Pamplin et al. 2005)

\section{Bothrioneurum iris Beddard, 1901}

Records: ecological remarks

São Paulo: leaf litter of Tietê River (Marcus 1942).

Bothrioneurum vejdovskyanum Stolc, 1886

Bothrioneurum pyrrhum, Marcus 1942: 201-205.

Records: ecological remarks

São Paulo: Tietê River, sediment (Marcus 1942).

Branchiura sowerbyi Beddard, 1892

Branchiura sowwerbyi, Alves \& Strixino 2003: 4; Dornfeld et al. 2006: 193; Fusari \& Fonseca-Gessner 2006: 93; Pamplin et al. 2006: 126 .

\section{Records: ecological remarks}

Luiz Antônio: Mogi-Guaçu River, sediment (Alves \& Strixino 2000, Alves \& Strixino 2003). Salesópolis and Bariri: Tietê River, Ponte Nova and Bariri Dams, sediment (Pamplin et al. 2005). Americana: Atibaia River, Salto Grande Dam, sediment (Dornfeld et al. 2006). Americana: Americana Dam, sediment (Pamplin et al. 2006). São Carlos: Monjolinho Dam, sediment (Fusari \& Fonseca-Gessner 2006). Barra Bonita: Tietê River, Barra Bonita Reservoir, sediment; Bariri: Tietê River, Bariri Reservoir, sediment; Ibitinga: Tietê River, Ibitinga Reservoir, sediment; Promissão: Tietê River, Promissão Reservoir, sediment; Buritama: Tietê River, Nova Avanhandava Reservoir, sediment and Pereira Barreto: Tietê River, Três Irmãos Reservoir, sediment (Suriani et al. 2007).

Chaetogaster diaphanus (Gruithuisen, 1828)

Records: ecological remarks

Araraquara: Cruzes Stream, associated with the gastropod Pomacea bridgesii (Gorni \& Alves 2006). Brotas: Lagoa Dourada Dam, associated with the sponge Metania spinata (Gorni \& Alves 2008a).

Chaetogaster diastrophus (Gruithuisen, 1828)

Records: ecological remarks 
São Paulo and Campinas: Tietê River sediment and associated with Bromeliaceae (Marcus 1943). Ipeúna: Cantagalo and Lapa Streams, associated with the Odonata larvae Elasmothemis cannacrioides and Mnesarete sp. (Corbi et al. 2004). Brotas: Lagoa Dourada Dam, associated with the sponge Metania spinata (Gorni \& Alves 2008a). Campos do Jordão: Galharada and Serrote Streams, sediment and leaf litter (Gorni \& Alves 2008b, Gorni \& Alves 2012).

\section{Chaetogaster langi Bretscher, 1896}

Chaetogaster parvus, Marcus 1943: 15.

Chaetogaster spongillae, Marcus 1943: 16.

Records: ecological remarks

São Paulo: Pinheiros River, sediment. Águas de São Pedro: Piracicaba River Basin, Araquá River sediment and Southern State of São Paulo: Ribeira de Iguape River, associated with the sponge Ephydatia crateriformis (Marcus 1943).

\section{Chaetogaster limnaei limnaei K. Von Baer, 1827}

Records: ecological remarks

São Paulo: associated with the gastropod Australorbis sp. (Du Bois-Reymond Marcus 1947).

\section{Dero botrytis Marcus, 1943}

Dero (Dero) botrytis, Pamplin et al. 2005: 67.

Records: ecological remarks

São Paulo: São Paulo University Campus (Marcus 1943). Águas de São Pedro: Piracicaba River Basin, sediment of Limoeiro Dam (Marcus 1944). Salesópolis and Bariri: Tietê River, Ponte Nova and Bariri Dams, sediment (Pamplin et al. 2005).

Dero digitata (O.F. Müller, 1773)

Dero (Dero) digitata, Alves \& De Lucca 2000: 115; Pamplin et al. 2005: 67; Gorni \& Alves 2006: 1060; Alves \& Gorni 2007: 409; Suriani et al. 2007: 420-421.

\section{Records: ecological remarks}

Araraquara: Pinheirinho and Santa Clara Streams, sediment (Alves \& De Lucca 2000). Salesópolis and Bariri: Tietê River, Ponte Nova and Bariri Dams, sediment (Pamplin et al. 2005) Araraquara: associated with the gastropod Pomacea bridgesii (Gorni \& Alves 2006). Brotas and Américo Brasiliense: Lagoa Dourada and Anhumas Stream Dams, associated with aquatic macrophytes (Alves \& Gorni 2007). Bariri: Tietê River, Bariri Reservoir, sediment (Suriani et al. 2007).

\section{Dero evelinae Marcus, 1943}

Dero (Dero) evelinae, Correia \& Trivinho-Strixino 1998: 41; Trivinho-Strixino et al. 2000: 533; Pamplin et al. 2005: 67; Fusari \& Fonseca-Gessner 2006: 93; Suriani et al. 2007: 420421; Alves \& Gorni 2007: 409.

\section{Records: ecological remarks}

São Paulo: University of São Paulo Campus (Marcus 1943). Luiz Antônio: Mogi-Guaçu River, associated with the aquatic macrophyte Scirpus cubensis (Correia \& Trivinho-Strixino 1998, Trivinho-Strixino et al. 2000). Salesópolis and Bariri: Tietê River, Ponte Nova and Bariri Dams, sediment (Pamplin et al. 2005). São Carlos: Fazzari Dam, sediment (Fusari \& Fonseca-Gessner 2006). Bariri: Tietê River, Bariri Reservoir, sediment (Suriani et al. 2007). Brotas and Américo Brasiliense: Lagoa Dourada and Anhumas Stream Dams, associated with aquatic macrophytes (Alves \& Gorni 2007).

\section{Dero multibranchiata Stieren, 1892}

Dero (Dero) multibranchiata, Corbi \& Trivinho-Strixino 2002: 38; Pamplin et al. 2005: 67; Suriani et al. 2007: 420-421.

\section{Records: ecological remarks}

Américo Brasiliense: Anhumas Stream Dam, sediment (Corbi \& Trivinho-Strixino 2002). Salesópolis and Bariri:
Tietê River, Ponte Nova and Bariri Dams, sediment (Pamplin et al. 2005). Bariri: Tietê River, Bariri Reservoir, sediment (Suriani et al. 2007).

\section{Dero nivea Aiyer, 1930}

Dero (Dero) nivea, Correia \& Trivinho-Strixino 1998: 41; Trivinho-Strixino et al. 2000: 533; Alves \& Strixino 2000: 177; Corbi \& Trivinho-Strixino 2002: 38; Pamplin et al. 2005: 67; Gorni \& Alves 2006: 1060; Suriani et al. 2007: 420-421; Alves \& Gorni 2007: 409; Gorni \& Alves 2008a: 262.

\section{Records: ecological remarks}

Luiz Antônio: Mogi-Guaçu River, associated with the aquatic macrophyte Scirpus cubensis (Correia \& TrivinhoStrixino 1998, Trivinho-Strixino et al. 2000), and sediment (Alves \& Strixino 2000). Américo Brasiliense: Anhumas Stream Dam, sediment (Corbi \& Trivinho-Strixino 2002). Salesópolis and Bariri: Tietê River, Ponte Nova and Bariri Dams, sediment (Pamplin et al. 2005). Araraquara: associated with the gastropod Pomacea bridgesii (Gorni \& Alves 2006). Barra Bonita: Tietê River, Barra Bonita Reservoir, sediment; Bariri: Tietê River, Bariri Reservoir, sediment (Suriani et al. 2007). Brotas and Américo Brasiliense: Lagoa Dourada and Anhumas Stream Dams, associated with aquatic macrophytes (Alves \& Gorni 2007). Brotas: Lagoa Dourada Dam, associated with the sponge Metania spinata (Gorni \& Alves 2008a).

\section{Dero obtusa D'Udekem, 1855}

Dero (Dero) obtusa, Alves \& De Lucca 2000: 115; Corbi \& Trivinho-Strixino 2002: 38; Pamplin et al. 2005: 67;Gorni \& Alves 2006: 1060; Suriani et al. 2007: 420-421; Alves \& Gorni 2007: 409; Gorni \& Alves 2008a: 262.

\section{Records: ecological remarks}

São Paulo: Pinheiros River tributaries, sediment (Marcus 1943). Araraquara: Pinheirinho and Santa Clara Streams, sediment (Alves \& De Lucca 2000). Américo Brasiliense: Anhumas Stream Dam, sediment (Corbi \& Trivinho-Strixino 2002). Salesópolis and Bariri: Tietê River, Ponte Nova and Bariri Dams, sediment (Pamplin et al. 2005). Araraquara: Cruzes Stream, associated with the gastropod Pomacea bridgesii (Gorni \& Alves 2006). Brotas and Américo Brasiliense: Lagoa Dourada and Anhumas Stream Dams, associated with aquatic macrophytes (Alves \& Gorni 2007). Barra Bonita: Tietê River, Barra Bonita Reservoir, sediment (Suriani et al. 2007). Brotas: Lagoa Dourada Dam, associated with the sponge Metania spinata (Gorni \& Alves 2008a).

\section{Dero pectinata Aiyer, 1930}

Dero (Dero) pectinata, Pamplin et al. 2005: 67; Suriani et al. 2007: 420-421.

Records: ecological remarks

Salesópolis and Bariri: Tietê River, Ponte Nova and Bariri Dams, sediment (Pamplin et al. 2005). Ibitinga: Tietê River, Ibitinga Reservoir, sediment (Suriani et al. 2007).

Dero plumosa Naidu, 1962

Dero (Dero) plumosa, Alves \& Strixino 2000: 177.

Records: ecological remarks

Luiz Antônio: Mogi-Guaçu River, sediment (Alves \& Strixino 2000).

Dero raviensis (Stephenson, 1914)

Dero (Dero) raviensis, Alves et al. 2006: 434; Gorni \& Alves 2006: 1060; Alves \& Gorni 2007: 409; Gorni \& Alves 2008a: 262.

\section{Records: ecological remarks}

Araraquara: Pinheirinho Stream, sediment and associated with the gastropod Pomacea bridgesii (Gorni \& Alves 2006). 
São Carlos: Monjolinho River, sediment (Alves et al. 2006). Brotas and Américo Brasiliense: Lagoa Dourada and Anhumas Stream Dams, associated with aquatic macrophytes (Alves \& Gorni 2007). Brotas: Lagoa Dourada Dam, associated with the sponge Metania spinata (Gorni \& Alves 2008a).

\section{Dero sawayai Marcus, 1943}

Dero (Dero) sawayai, Gorni \& Alves 2006: 1060; Alves \& Gorni 2007: 409; Gorni \& Alves 2008a: 262.

\section{Records: ecological remarks}

São Paulo: Pinheiros River tributaries, sediment (Marcus 1943). Araraquara: associated with the gastropod Pomacea bridgesii (Gorni \& Alves 2006). Brotas and Américo Brasiliense: Lagoa Dourada and Anhumas Stream Dams, associated with aquatic macrophytes (Alves \& Gorni 2007). Brotas: Lagoa Dourada Dam, associated with the sponge Metania spinata (Gorni \& Alves 2008a).

\section{Haemonais waldvogeli Bretscher, 1900}

Records: ecological remarks

Salesópolis and Bariri: Tietê River, Ponte Nova and Bariri Dams, sediment (Pamplin et al. 2005, Suriani et al. 2007). Araraquara: Pinheirinho Stream, sediment (Alves et al. 2006), and associated with the gastropod Pomacea bridgesii (Gorni \& Alves 2006).

\section{Jolydrilus jaulus Marcus, 1965}

Records: ecological remarks

Cananéia and Cubatão: mangrove sediment (Marcus 1965).

\section{Limnodrilus hoffmeisteri Claparède, 1862}

Limnodrilus hoffmeisteri f. divergens, Marcus 1942: 169-174. Limnodrilus hoffmeisteri f. parva, Marcus 1942: 167-169.

\section{Records: ecological remarks}

São Paulo: Tietê River, sediment (Marcus 1942). Pirituba (Mendes et al. 1951). Luiz Antônio: Mogi-Guaçu River, sediment (Alves \& Strixino 2000, Alves \& Strixino 2003). Araraquara: Pinheirinho, Santa Clara and àgua Branca Streams, sediment (Alves \& De Lucca 2000, Alves et al. 2006). Salesópolis and Bariri: Tietê River, Ponte Nova and Bariri Dams, sediment (Pamplin et al. 2005). São Carlos: Monjolinho River, sediment (Alves et al. 2006, Fusari \& Fonseca-Gessner 2006). Ribeirão Preto: Pardo River, sediment of Monte Alegre Lake (Cleto-Filho \& Arcifa 2006). Americana: Atibaia River, Salto Grande Dam, sediment (Dornfeld et al. 2006). Americana: Americana Dam, sediment (Pamplin et al. 2006). Barra Bonita: Tietê River, Barra Bonita Reservoir, sediment; Bariri: Tietê River, Bariri Reservoir, sediment. Ibitinga: Tietê River, Ibitinga Reservoir, sediment; Promissão: Tietê River, Promissão Reservoir, sediment; Buritama: Tietê River, Nova Avanhandava Reservoir, sediment and Pereira Barreto: Tietê River, Três Irmãos Reservoir, sediment (Suriani et al. 2007). Ribeirão Grande: Intervales State Park Streams (Alves et al. 2008). Campos do Jordão: Galharada and Serrote Streams, sediment and leaf litter (Gorni \& Alves 2008b, Gorni \& Alves 2012).

\section{Limnodrilus neotropicus Černosvitov, 1939}

Records: ecological remarks

Araraquara: Água Branca Stream, sediment (Alves et al. 2006). São Carlos: Gouveia Stream, sediment (Alves et al. 2006). Ribeirão Grande: Intervales State Park Streams (Alves et al. 2008).

Limnodrilus udekemianus Claparède, 1862

Records: ecological remarks

São Paulo: Tietê River, sediment (Marcus 1942). Salesópolis and Bariri: Tietê River, Ponte Nova and Bariri
Dams, sediment (Pamplin et al. 2005). São Carlos: Monjolinho River, sediment (Alves et al. 2006). Buritama: Tietê River, Nova Avanhandava Reservoir, sediment (Suriani et al. 2007). Pereira Barreto: Tietê River, Três Irmãos Reservoir, sediment (Suriani et al. 2007).

\section{Monopylephorus parvus Ditlevsen, 1904}

Records: ecological remarks

Cananéia: mangrove sediment (Marcus 1965).

Nais communis Piguet, 1906

Nais communis f. magenta, Marcus 1943: 23.

Records: ecological remarks

Southern State of São Paulo: Ribeira de Iguape River, associated with the sponge Ephydatia crateriformis (Marcus 1943). São Paulo: Tietê River and Pinheiros River tributaries, sediment (Marcus 1943). Luiz Antônio: Mogi-Guaçu River, associated with aquatic macrophytes (Trivinho-Strixino et al. 2000). Araraquara: Pinheirinho and Santa Clara Streams, sediment (Alves \& De Lucca 2000), and associated with the gastropod Pomacea bridgesii (Gorni \& Alves 2006). São Carlos: Jacaré-Guaçu River, associated with the sponge Radiospongilla amazonensis (Corbi et al. 2005), and Monjolinho River, sediment (Alves et al. 2006). Brotas and Américo Brasiliense: Lagoa Dourada and Anhumas Stream Dams, associated with aquatic macrophytes (Alves \& Gorni 2007). Brotas: JacaréPepira River, associated with the bryophytes Fissidens sp. and Philonotis sp. (Gorni \& Alves 2007), and Lagoa Dourada Dam, associated with the sponge Metania spinata (Gorni \& Alves 2008a). Ribeirão Grande: Intervales State Park Streams (Alves et al. 2008). Campos do Jordão: Campo do Meio and Galharada Streams, sediment and leaf litter (Gorni \& Alves 2008b, Gorni \& Alves 2012).

Nais elinguis O. F. Müller, 1774

Records: ecological remarks

Araraquara: associated with the gastropod Pomacea bridgesii (Gorni \& Alves 2006).

\section{Nais pardalis Piguet, 1906}

Records: ecological remarks

São Paulo: Pinheiros River tributaries, sediment (Marcus 1943). Araraquara: associated with the gastropod Pomacea bridgesii (Gorni \& Alves 2006).

Nais variabilis Piguet, 1906

Records: ecological remarks

Ipeúna: Cantagalo and Lapa Streams, associated with the Odonata larvae Elasmothemis cannacrioides and Mnesarete sp. (Corbi et al. 2004). Brotas and Américo Brasiliense: Lagoa Dourada and Anhumas Stream Dams, associated with aquatic macrophytes (Alves \& Gorni 2007). Campos do Jordão: Galharada Stream, sediment and leaf litter (Gorni and Alves 2008b, Gorni \& Alves 2012).

Paranais evelinae (Marcus, 1965)

Wapsa evelinae, Marcus 1965: 63.

Records: ecological remarks

Cananéia: mangrove sediment (Marcus 1965).

Pristina aequiseta aequiseta Bourne, 1891

Pristina aequiseta, Marcus 1943: 104-106, Alves et al. 2006: 434, Gorni \& Alves 2008a: 262.

Pristina evelinae, Marcus 1943: 112-125.

Records: ecological remarks

São Paulo: Pinheiros River tributaries, sediment; Pirassununga: Mogi-Guaçu River, Cachoeira de Emas Reservoir, sediment and Águas de São Pedro: Piracicaba River Basin, Araquá River, sediment (Marcus 1943). Southern 
State of São Paulo: Ribeira de Iguape River, associated with the sponge Ephydatia crateriformis (Marcus 1943). Araraquara: Água Branca Stream, sediment (Alves et al. 2006). Brotas: Lagoa Dourada Dam, associated with the sponge Metania spinata (Gorni \& Alves 2008a).

Pristina americana Cernosvitov, 1937

Pristinella longidentata, Alves \& Strixino 2000: 177; Gorni \& Alves 2008b: 163.

Pristina (Pristinella) longidentata, Gorni \& Alves 2012: 107.

Records: ecological remarks

São Paulo: Pinheiros and Tietê Rivers, sediment (Marcus 1943). Luiz Antônio: Mogi-Guaçu River, sediment (Alves \& Strixino 2000). Araraquara: Pinheirinho and Santa Clara Streams, sediment (Alves \& De Lucca 2000). Salesópolis and Bariri: Tietê River, Ponte Nova and Bariri Dams, sediment (Pamplin et al. 2005). São Carlos: Monjolinho River, sediment (Alves et al. 2006). Barra Bonita: Tietê River, Barra Bonita Reservoir, sediment; Bariri: Tietê River, Bariri Reservoir, sediment; Ibitinga: Tietê River, Ibitinga Reservoir, sediment; Promissão: Tietê River, Promissão Reservoir, sediment; Buritama: Tietê River, Nova Avanhandava Reservoir, sediment and Pereira Barreto: Tietê River, Três Irmãos Reservoir, sediment (Suriani et al. 2007). Campos do Jordão: Galharada Stream, sediment and leaf litter (Gorni \& Alves 2008b).

\section{Pristina biserrata Chen, 1940}

\section{Records: ecological remarks}

Brotas and Américo Brasiliense: Lagoa Dourada and Anhumas Stream Dams, associated with aquatic macrophytes (Alves \& Gorni 2007), and with the sponge Metania spinata (Gorni \& Alves 2008a). Campos do Jordão: Galharada Stream, sediment and leaf litter (Gorni \& Alves 2008b).

Pristina breviseta Bourne, 1891

Naidium breviseta, Marcus 1943: 128-129.

Records: ecological remarks

São Paulo: Tietê River, sediment (Marcus 1943). Américo Brasiliense: Anhumas Stream Dam, sediment (Corbi \& Trivinho-Strixino 2002). São Carlos: Fazzari Dam, sediment (Fusari \& Fonseca-Gessner 2006).

\section{Pristina longiseta Ehrenberg, 1828}

Pristina leidyi, Alves \& Strixino 2003: 4; Gorni \& Alves 2006: 1060; Alves \& Gorni 2007: 409; Gorni \& Alves 2008a: 262; Gorni \& Alves 2008b: 163; Gorni \& Alves 2012: 107.

\section{Records: ecological remarks}

Southern State of São Paulo: Ribeira de Iguape River, associated with the sponge Ephydatia crateriformis, and Águas de São Pedro: Piracicaba River Basin, Araquá River, sediment (Marcus 1943). Luiz Antonio: Mogi-Guaçu River, sediment (Alves \& Strixino 2003). Araraquara: associated with the gastropod Pomacea bridgesii (Gorni \& Alves 2006). Brotas and Américo Brasiliense: Lagoa Dourada and Anhumas Stream Dams, associated with aquatic macrophytes (Alves \& Gorni 2007), and with the sponge Metania spinata (Gorni \& Alves 2008a). Campos do Jordão: Galharada Stream, sediment and leaf litter (Gorni \& Alves 2008b, Gorni \& Alves 2012).

Pristina macrochaeta Stephenson, 1931

Records: ecological remarks

São Paulo: Pinheiros River, sediment (Marcus 1943). Campinas: Atibaia River, sediment (Marcus 1944). Brotas and Américo Brasiliense: Lagoa Dourada and Anhumas Stream Dams, associated with aquatic macrophytes (Alves \& Gorni 2007), and with the sponge Metania spinata (Gorni \& Alves 2008a).
Pristina menoni (Aiyer, 1929)

Pristinella menoni, Alves et al. 2006: 434; Gorni \& Alves 2007: 519.

Records: ecological remarks

Araraquara: Pinheirinho Stream, sediment (Alves et al. 2006). Brotas: Jacaré-Pepira River, associated with the bryophytes Fissidens sp. and Philonotis sp. (Gorni \& Alves 2007).

Pristina notopoda Černosvitov, 1937

Pristinella notopora, Gorni \& Alves 2008b: 163.

Pristina (Pristinella) notopora, Gorni \& Alves 2012: 107.

Records: ecological remarks

Campos do Jordão: Campo do Meio, Galharada and Serrote Streams, sediment and leaf litter (Gorni \& Alves 2008b, Gorni \& Alves 2012).

Pristina osborni (Walton, 1906)

Naidium minutum, Marcus 1943: 129-130.

Pristinella osborni, Alves \& Gorni 2007: 409; Alves et al. 2008: 71 .

Pristinella minuta, Gorni \& Alves 2008b: 163.

Pristina (Pristinella) minuta, Gorni \& Alves 2012: 107.

Records: ecological remarks

Águas de São Pedro: Piracicaba River Basin, Araquá River, sediment (Marcus 1943). Brotas and Américo Brasiliense: Lagoa Dourada and Anhumas Stream Dams, associated with aquatic macrophytes (Alves \& Gorni 2007). Ribeirão Grande: Intervales State Park Streams (Alves et al. 2008). Campos do Jordão: Galharada Stream, sediment and leaf litter (Gorni \& Alves 2008b, Gorni \& Alves 2012).

\section{Pristina proboscidea Beddard, 1896}

Records: ecological remarks

São Carlos: Jacaré-Guaçu River, associated with the sponge Radiospongilla amazonensis (Corbi et al. 2005). Araraquara: Água Branca Stream, sediment (Alves et al. 2006). Brotas and Américo Brasiliense: Lagoa Dourada and Anhumas Stream Dams, associated with aquatic macrophytes (Alves \& Gorni 2007). Brotas: Lagoa Dourada Dam, associated with the sponge Metania spinata (Gorni \& Alves 2008a). Campos do Jordão: Galharada Stream, sediment and leaf litter (Gorni \& Alves 2008b, Gorni \& Alves 2012).

\section{Pristina rosea (Piguet, 1906)}

Naidium roseum, Marcus 1943: 130-132; Marcus 1944: 50.

Pristinella jenkinae, Alves et al. 2006: 434; Fusari \& Fonseca-Gessner 2006: 93; Gorni \& Alves 2007: 519; Gorni \& Alves 2008b: 163; Gorni \& Alves 2012: 107.

\section{Records: ecological remarks}

São Paulo: University of São Paulo Campus, and Pinheiros River tributaries, sediment (Marcus 1943), Tietê River, associated with the plant Calathea sp. (Marcus 1944). Araraquara: Pinheirinho and Água Branca Streams, sediment (Alves et al. 2006). São Carlos: Gouveia Stream, sediment (Alves et al. 2006), and Monjolinho Dam, sediment (Fusari \& Fonseca-Gessner 2006). Brotas: Jacaré-Pepira River, associated with the bryophytes Fissidens sp. and Philonotis sp. (Gorni \& Alves 2007). Campos do Jordão: Campo do Meio Stream, Galharada and Serrote Streams, leaf litter and sediment (Gorni \& Alves 2008b, Gorni \& Alves 2012).

Pristina sima (Marcus, 1944)

Naidium simum, Marcus 1944: 68-69.

Pristinella sima, Gorni \& Alves 2008b: 163.

Pristina (Pristinella) sima, Gorni \& Alves 2012: 107.

Records: ecological remarks 
São Paulo: associated with the bryophyte Dumortiera sp. (Marcus 1944). Luiz Antônio: Mogi-Guaçu River, sediment (Alves \& Strixino 2000). Campos do Jordão: Galharada and Serrote Streams, leaf litter and sediment (Gorni \& Alves 2008b, Gorni \& Alves 2012).

Pristina synclites Stephenson, 1925

\section{Records: ecological remarks}

Araraquara: Pinheirinho Stream, sediment (Alves et al. 2006). São Carlos: Monjolinho River, sediment (Alves et al. 2006, Fusari \& Fonseca-Gessner 2006).

\section{Slavina appendiculata (D’Udekem, 1855)}

Records: ecological remarks

São Carlos: Gouveia Stream, sediment (Alves et al. 2006).

Slavina evelinae (Marcus, 1942)

Peloscolex evelinae, Marcus 1942: 157-159.

Records: ecological remarks

São Paulo: Tietê River, sediment (Marcus 1942). Luiz Antônio: Mogi-Guaçu River, associated with the aquatic macrophyte Scirpus cubensis (Correia \& Trivinho-Strixino 1998, Trivinho-Strixino et al. 2000). Américo Brasiliense: Anhumas Stream Dam, sediment (Corbi \& Trivinho-Strixino 2002). Salesópolis and Bariri: Tietê River, Ponte Nova and Bariri Dams, sediment (Pamplin et al. 2005, Suriani et al. 2007). Brotas and Américo Brasiliense: Lagoa Dourada and Anhumas Stream Dams, associated with aquatic macrophytes (Alves \& Gorni 2007).

Slavina isochaeta Černosvitov, 1939

Records: ecological remarks

Luiz Antonio: Mogi-Guaçu River, sediment (Alves \& Strixino 2003).

Slavina sawayai Marcus, 1944

Records: ecological remarks

Águas de São Pedro: Piracicaba River Basin, Araquá River, sediment (Marcus 1944).

Stylaria lacustris (Linnaeus, 1767)

Records: ecological remarks

Brotas and Américo Brasiliense: Lagoa Dourada and Anhumas Stream Dams, associated with aquatic macrophytes (Alves \& Gorni 2007).

Tectidrilus gabriellae Du Bois-Reymond Marcus, 1950

Peloscolex gabriellae, Du Bois-Reymond Marcus 1950: 1.

Records:

São Sebastião (Du Bois-Reymond Marcus 1950).

Tubifex tubifex tubifex (O. F. Müller, 1774)

Tubifex (Tubifex) tubifex, Marcus 1942: 198-200.

Tubifex tubifex, Alves \& Strixino 2000: 177; Alves et al. 2006: 434.

Records: ecological remarks

São Paulo: Tietê River, sediment (Marcus 1942). Luiz Antônio: Mogi-Guaçu River, sediment (Alves \& Strixino 2000). São Carlos: Monjolinho River, sediment (Alves et al. 2006).

\section{Narapidae}

Narapa bonettoi Righi \& Varela, 1983

Records: ecological remarks

São Carlos: Gouveia Stream, sediment (Alves et al. 2006). OPISTOCYSTIDAE

Opistocysta funiculus Cordero, 1948

Opistocysta flagellum, Marcus 1944: 69-70.

Records: ecological remarks

São Paulo: Tietê River, sediment (Marcus 1944). Luiz Antônio: Mogi-Guaçu River, sediment (Alves \& Strixino 2000, Alves \& Strixino 2003). Américo Brasiliense: Anhumas Stream
Dam, sediment (Corbi \& Trivinho-Strixino 2002). Salesópolis and Bariri: Tietê River, Ponte Nova and Bariri Dams, sediment (Pamplin et al. 2005). Americana: Atibaia River, Salto Grande Dam, sediment (Dornfeld et al. 2006), and in Americana Dam (Pamplin et al. 2006). Barra Bonita: Tietê River, Barra Bonita Reservoir, sediment; Ibitinga: Tietê River, Ibitinga Reservoir, sediment; Buritama: Tietê River, Nova Avanhandava Reservoir, sediment; Pereira Barreto: Tietê River, Três Irmãos Reservoir, sediment (Suriani et al. 2007).

\section{References}

ALVES, R.G. \& GORNI, G.R. 2007. Naididae species (Oligochaeta) associated with submersed aquatic macrophytes in two reservoirs (São Paulo, Brazil). Acta Limnol. Bras. 19(4):407-413.

ALVES, R.G. \& LUCCA, J.V. 2000. Oligochaeta (Annelida: Clitellata) como indicador de poluição orgânica em dois córregos pertencentes à Bacia do Ribeirão do Ouro - Araraquara (São Paulo-Brasil). Braz. J. Ecol. 4(1-2):112-117.

ALVES, R.G. \& STRIXINO, G. 2000. Distribuição espacial de Oligochaeta em uma lagoa marginal do rio Mogi-GuaçuSP. Iheringia, Sér. Zool. 88:173-180.

ALVES, R.G. \& STRIXINO, G. 2003. The sampling of benthic macroinvertebrates using two different methods: Waiting trays and an Ekman collector. Acta Limnol. Bras. 15(3):1-6.

ALVES, R.G., MARCHESE, M.R. \& ESCARPINATI, S.C. 2006. Oligochaeta (Annelida, Clitellata) in lotic environments in the state of São Paulo, Brazil. Iheringia, Sér. Zool. 96(4):431-435, http:// dx.doi.org/10.1590/S0073-47212006000400007

ALVES, R.G., MARCHESE, M.R \& MARTINS, R.T. 2008. Oligochaeta (Annelida, Clitellata) of lotic environments at Parque Estadual Intervales (São Paulo, Brasil). Biota Neotrop. 8(1):69-72, http://dx.doi.org/10.1590/S1676-06032008000100009

BRINKURST, R.O. \& MARCHESE, M.R. 1989. Guia para la identificación de oligoquetos acuáticos continentales de Sud y Centroamerica Santa Fé: Asociación de ciencias naturales del litoral.

CHRISTOFFERSEN, M.L. 2007. A catalogue of aquatic microdrile oligochaetes (Annelida: Clitellata) from South America. Acta Hydrobiol. Sin. 31:59-86.

CLETO-FILHO, S.E.N. \& ARCIFA, M.S. 2006. Horizontal distribution and temporal variation of the zoobenthos of a tropical Brazilian lake. Acta Limnol. Bras. 18(4):407-421.

CORBI, J.J. \& TRIVINHO-STRIXINO, S.2002. Spatial and bathymetric distribution macrobenthic fauna of the Ribeirão das Anhumas reservoir (Américo Brasiliense-SP, Brasil). Acta Limnol. Bras. 10(1):37-47.

CORBI, J.J., JANCSO, M.A. \& TRIVINHO-STRIXINO, S., FRAGOSO, E.N. 2004. Occurence of Oligochaeta living on larvae of Odonata from ipeúna (São Paulo state, Brazil). Biota Neotrop. 4(2):1-3, http://dx.doi.org/10.1590/S1676-06032004000200017

CORBI, J.J., TRIVINHO-STRIXINO, S. \& ALVES, R.G. 2005. Records of oligochaetes in freshwater sponges, on bryozoarians and on colonial hydrozoans from Brazil. Braz. J. Biol. 65(1):187188, http://dx.doi.org/10.1590/S1519-69842005000100022

CORREIA， L.C.S. \& TRIVINHO-STRIXINO, S. 1998. Macroinvertebrados da Rizosfera de Scirpus cubensis na lagoa do Infernão (Estação Ecológica de Jataí - SP): Estrutura e função. Acta Limnol. Bras. 10(1):37-47.

DORNFELD, C.B., ALVES, R.G., LEITE, M.A. \& ESPÍNDOLA, E.L.G. 2006. Oligochaeta in eutrofic reservoir: the case of Salto Grande reservoir and their main affluent (Americana, São Paulo, Brazil). Acta Limnol. Bras. 18(2):189-197.

DU BOIS-REYMOND MARCUS, E. 1944. Notes on fresh-water Oligochaeta from Brazil. Comun. Zool. Mus. Hist. Nat. Montev. $1: 1-8$ 
DU BOIS-REYMOND MARCUS, E. 1947. Naidids and tubificids from Brazil. Comun. Zool. Mus. Hist. Nat. Montev. 2(44):1-20.

DU BOIS-REYMOND MARCUS, E. 1950. A marine tubificid from Brazil. Comun. Zool. Mus. Hist. Nat. Montev. 3(59):1-6.

FUSARI, L.M. \& FONSECA-GESSNER, A.A. 2006. Environmental assessment of two small reservoirs in southeastern Brazil, using macroinvertebrate community metrics. Acta Limnol. Bras. 18(1):89-99.

GORNI, G.R. \& ALVES, R.G. 2006. Naididae (Annelida, Oligochaeta) associated with Pomacea bridgesii (Reeve) (Gastropoda, Ampullaridae). Rev. Bras. Zool. 23(4):1059-1061, http://dx.doi.org/10.1590/S0101-81752006000400011

GORNI, G.R. \& ALVES, R.G. 2007. Naididae (Annelida, Oligochaeta) associated with briophytes in Brotas, State of São Paulo, Brazil. Rev. Bras. Zool. 24(2):518-519, http://dx.doi.org/ 10.1590/S0101-81752007000200036

GORNI, G.R. \& ALVES, R.G. 2008a. Naididae species (Annelida: Oligochaeta) associated with the sponge Metania spinata (Carter, 1881) (Porifera: Metaniidae) from a southeastern reservoir. Acta Limnol. Bras. 20(3):261-263.

GORNI, G.R. \& ALVES, R.G. 2008b. Oligochaeta (Annelida: Clitellata) em córregos de baixa ordem do Parque Estadual de Campos do Jordão (São Paulo - Brasil). Biota Neotrop. 8(4):161165, http://dx.doi.org/10.1590/S1676-06032008000400016

GORNI, G.R. \& ALVES, R.G. 2012. Oligochaetes (Annelida, Clitellata) in a neotropical stream: a mesohabitat approach. Iheringia, Sér. Zool. 102(1):106-110.

JANKE, H. \& TRIVINHO-STRIXINO, S. 2007. Colonization of leaf litter by aquatic macroinvertebrates: a study in a low order tropical stream. Acta Limnol. Bras. 19(1):109-115 2007.

LAFONT, M. 1984. Oligochaete communities as biological descriptors of pollution in the fine sediments of rivers. Hydrobiologia 115:127129, http://dx.doi.org/10.1007/BF00027906

MARCHESE, M. \& DRAGO, I.E. 1999. Use of benthic macroinvertebrates as organic pollution indicators in lotic enviroments of Paraná River drainage basin. Pol. Arch. Hydrobiol. 46(3-4):233255.

MARCUS, E. 1942. Sôbre algumas Tubificidae do Brasil. Bol. Fac. Fil. Ci. Letr. Univ. S. Paulo. 25(6):153-228.

MARCUS, E. 1943. Sobre Naididae do Brasil. Bol. Fac. Fil. Ci. Letr. Univ. S. Paulo. 32(7):3-247.

MARCUS, E. 1944. Sobre Oligochaeta límnicos do Brasil. Bol. Fac. Fil. Ci. Letr. Univ. S. Paulo. 43(8):5-135.

MARCUS, E. 1965. Naidomorpha aus brasilianischem Brackwasser. Beiträge für Neotropischen Fauna. 4:61-83, http://dx.doi.org/ 10.1080/01650526509360380

MARTIN, P., MARTÍNEZ-ANSEMIL, E., PINDER, A., TIMM, T. \& WETZEL, M.J. 2008. Global diversity of oligochaetous clitellates (Oligochaetä: Clitellata) in freshwater. In: Freshwater animal diversity assessment (Balian, E.V., Lévêque, C., Segers, H., Martens, K., ). Hydrobiologia vol. 595, pars 1, p.117-127.

MARTINS, R.T. \& ALVES, R.G. 2010. Occurrence of Chaetogaster limnaei K. von Baer, 1927 (Oligochaeta, Naididae) associated with Gastropoda mollusks in horticultural channels in Southeastern
Brazil. Braz. J. Biol. (Impresso) 70:1055-1057, http://dx.doi.org/ 10.1590/S1519-69842010000500020

MENDES, E.G., PÉREZ GONZÁLEZ, M.D \& COUTINHO, M.L. 1951. On the function of hemoglobine in limnic Oligochaeta. Bol. Fac. Fil. Ci. Letr. Univ. S. Paulo. 16:289-301.

MONTANHOLI-MARTINS, M.C. \& TAKEDA, A.M. 1999. Communities of benthic oligochaetes in relation to sediment structure in the Upper Paraná River, Brazil. Stud. Neotrop. Fauna Environ. 34:52-58, http://dx.doi.org/10.1076/snfe.34.3.52. 8899

MONTANHOLI-MARTINS, M.C. \& TAKEDA, A.M. 2001. Spatial and temporal variations of oligochaetes of the Ivinhema River and Patos Lake in the Upper Paraná River Basin, Brazil. Hydrobiologia 463(1-3):197-205, http://dx.doi.org/10.1023/A:1013163927814

PAMPLIN, P.A.Z., ROCHA, O. \& MARCHESE, M. 2005. Riqueza de espécies de Oligochaeta (Anellida, Clitellata) em duas represas do Rio Tietê (São Paulo). Biota Neotrop. 5(1):1-8, http:// dx.doi.org/10.1590/S1676-06032005000100007

PAMPLIN, P.A.Z., ALMEIDA, T.C.M. \& ROCHA, O. 2006. Composition and distribution of benthic macroinvertebrados in Americana Reservoir (SP, Brazil). Acta Limnol. Bras. 18(2):121132.

RIGHI, G. 1984. Manual de identificação de invertebrados límnicos do Brasil. CNpq/Coordenação Editorial.

RIGHI, G. 2002. Anelídeos Oligoquetos. In: Biodiversidade do Estado de São Paulo, Brasil. Invertebrados de água doce. São Paulo: FAPESP. vol. 4, p.82-84.

RODRIGUES, L.F.T., LEITE, F.S. \& ALVES, R.G. 2013a. Inventory and distribution of Oligochaeta (Annelida, Clitellata) in first-order streams in preserved areas of the state of Minas Gerais, Brazil. Biota Neotrop. (Edição em Português. Online) 13:1-10.

RODRIGUES, L.F.T., SANTANA, L.D. \& ALVES, R.G. 2013 b. Aquatic oligochaetes associated with bryophytes in an Atlantic Forest stream. Biota Neotrop. (Edição em Português. Online) 3:1-6.

SURIANI, A.L., FRANÇA, R.S., PAMPLIN, P.A.Z., MARCHESE, M., LUCCA, J.V. \& ROCHA, O. 2007. Species richness and distribution of oligochaetes in six reservoirs on Middle and Low Tietê River (SP, Brazil). Acta Limnol. Bras. 19(4):415-426.

TAKEDA, A.M. 1999. Oligochaeta community of alluvial Upper Paraná River, Brazil: spatial and temporal distribution (1987-1988). Hydrobiologia 412:35-42, http://dx.doi.org/10.1023/A:1003844 131148

TAKEDA, A.M. 2001. Effect of hydraulics, bed load grain size and water factor on habitat and abundance of Narapa bonetoi Righi \& Varela, 1983 of the Upper Paraná River, Brazil. Oligochaeta community of alluvial Upper Paraná River, Brazil: spatial and temporal distribution (1987-1988). Hydrobiologia 463:35-42.

TIMM, T., SEIRE, A. \& PALL, P. 2001. Half a century of oligochaete research in Estonian running waters. Hydrobiologia 463:223-234, http://dx.doi.org/10.1023/A:1013176229631

TRIVINHO-STRIXINO, S., CORREIA, L.C.S. \& SONODA, K. 2000. Phytophilous Chironomidae (Diptera) and other macroinvertebrates in the ox-bow Infernão Lake (Jataí Ecological Station, Luiz Antônio, SP, Brazil). Rev. Bras. Biol. 60(3):527-535, http://dx.doi.org/10.1590/S0034-71082000000300018 